\title{
Photodynamic Therapy of Oral Dysplasia With Topical 5-Aminolevulinic Acid and Light-Emitting Diode Array
}

\author{
Jui-Chang Tsai, MD, PhD, ${ }^{1,2}$ Chun-Pin Chiang, BDS, DMse, ${ }^{3}$ Hsin-Ming Chen, BDs, DMSc, ${ }^{3}$ Sheng Bang Huang, Ms, ${ }^{4}$ \\ Chun Wei Wang, Ms, ${ }^{4}$ Ming I. Lee, $\mathrm{MD}^{5}{ }^{5}$ Yih-Chih Hsu, PhD, ${ }^{5}$ Chin-Tin Chen, PhD, ${ }^{2,6 * *}$ and Tsuimin Tsai, PhD ${ }^{7 *}$ \\ ${ }^{1}$ Division of Neurosurgery, Department of Surgery, National Taiwan University Hospital, Taipei 100, Taiwan \\ ${ }^{2}$ Center for Optoelectronic Biomedicine and College of Medicine, National Taiwan University, Taipei 100, Taiwan \\ ${ }^{3}$ School of Dentistry, College of Medicine, National Taiwan University, Taipei 100, Taiwan \\ ${ }^{4}$ Opto-Electronics \& Systems Laboratories, Industrial Technology Research Institute, Hsinchu 310, Taiwan \\ ${ }^{5}$ Biomedical Engineering Center, Industrial Technology Research Institute, Hsinchu 310, Taiwan \\ ${ }^{6}$ Graduate Institute of Oral Biology, College of Medicine, National Taiwan University, Taipei 100, Taiwan \\ ${ }^{7}$ Graduate Institute of Biomedical Materials, Taipei Medical University, Taipei 110, Taiwan
}

Background and Objectives: In Taiwan, more than two million people have the betel quid (BQ) chewing habit which is a risk factor related to premalignant lesion and squamous cell carcinoma of oral cavity. We developed a light-emitting diode (LED) array combined with topical 5aminolevulinic acid (ALA) for photodynamic therapy (PDT) and evaluated its effectiveness for the treatment of oral lesions.

Study Design/Materials and Methods: We compared the ALA-PDT effect of the homemade LED array to that of a commercial light source on cultured Ca9-22 human gingival carcinoma cells and the DMBA-induced hamster buccal pouch carcinoma model. Furthermore, we treated several patients having an oral lesion using a topical ALA delivery system and the LED array.

Results: The LED array light source was as effective as the commercial light source for ALA-PDT in cultured Ca9-22 cells with $\mathrm{LD}_{50}$ of 4.5 and $4.3 \mathrm{~J} / \mathrm{cm}^{2}$, respectively, using an MTT assay. This light source was also effective in the DMBA-induced hamster buccal pouch carcinoma model, and in the patients of oral leukoplakia.

Conclusions: ALA-PDT is effective for premalignant lesions such as mucosal dysplasia and carcinoma in situ of oral cavity. Good results could be obtained by using the homemade LED array as light source. The LED array has the advantages of low cost, high reliability, and portability. It is safe, convenient and easy to use for the treatment of oral dysplasia. Lasers Surg. Med. 34:18-24, 2004.

(c) 2004 Wiley-Liss, Inc.

Key words: light-emitting diode; 5 -aminolevulinic acid; photodynamic therapy; oral leukoplakia

\section{INTRODUCTION}

Oral cancer ranks as the seventh leading cause of death from cancers in Taiwan [1]. Its annual rate of increase was highest in 2001 among male patients, according to the cancer registry annual report published by the Department of Health, Taiwan. The increasing prevalence of oral cancer is probably related to the popular betel quid (BQ) chewing habit in this area $[2,3]$. More than two million people have this BQ chewing habit in Taiwan and approximately $80 \%$ of oral cancers are associated with this habit $[4,5]$. Oral leukoplakia is a white patch or plaque of oral mucosa, which is difficult to be characterized clinically or pathologically as any other definable disease. It is regarded as an oral premalignant lesion with a high risk for development of oral cancer [6]. In Taiwan, the development of oral leukoplakia is strongly associated with BQ chewing [7]. Thus, management of these precancerous oral lesions is a considerable problem. Currently, oral cancers are treated by a combination of surgery, radiation therapy, and chemotherapy. In spite of the combination therapy, the overall survival rates have not improved substantially in the last two decades [8]. Moreover, these aggressive treatments often cause difficulties in chewing, swallowing, speech, and even loss of the esthetics that makes the patients or their families very frustrated. Therefore, an effective therapeutic tool without the disadvantages of conventional modality is required in order to improve the treatment results of oral cancers.

Photodynamic therapy (PDT) has been developed as a modality for cancer treatment. It is based on the administration of photosensitizers (PS) which are selectively retained in tumor tissues and induce cytotoxicity after light irradiation $[9,10]$. This technique is based on the administration of exogenous photosensitizing drugs to render tumor tissue sensitive to non-thermal light of a specific wavelength. The sensitizers are normally inert and have a selective affinity to tumor tissues. In the presence of oxygen, light illumination activates the drug and in turn

\footnotetext{
Contract grant sponsor: National Taiwan University Hospital; Contract grant number: NTUH.90A06; Contract grant sponsor: The National Science Council, Taiwan; Contract grant number: NSC 89-2736-L-002-003.

${ }^{*}$ Correspondence to: Dr. Tsuimin Tsai, Graduate Institute of Biomedical Materials, Taipei Medical University, $250 \mathrm{Wu}-\mathrm{H}$ sing Street, Taipei 110, Taiwan. E-mail: tmtsai00@tmu.edu.tw

**Center for Optoelectronic Biomedicine, College of Medicine, National Taiwan University, No 1, Jen-Ai Road, Section 1st, Taipei 100, Taiwan. E-mail: ctchen@ha.mc.ntu.edu.tw Accepted 20 October 2003

Published online in Wiley InterScience

(www.interscience.wiley.com).

DOI 10.1002/lsm.10250
} 
produces singlet oxygen which induces a direct cellular killing through the type II photochemical reaction $[11,12]$. Besides, PDT may cause tumor cell death indirectly by damaging tumor-associated vasculature or activating host immune responses [13].

5-Aminolevulinic acid (ALA) has been successfully used in the diagnosis and treatment of neoplastic tissue [14]. ALA itself is not a PS and serves as the biological precursor in the heme biosynthetic pathway [15]. Administration of ALA bypasses the feedback control system in the heme biosynthetic pathway, resulting in cellular accumulation of protoporphyrin IX (PpIX, the PS). The potential usefulness of ALA-PDT in the treatment of tumors has been demonstrated in animal $[16,17]$ and human studies [18-20]. The side effect of cutaneous photosensitivity is commonly found in the clinical trials of PDT. Compared to other PS such as Photofrin ${ }^{\circledR}$, ALA-PDT has less prolonged photosensitivity or cumulative toxicity [21]. This is especially good for the treatment of oral precancerous or cancerous lesions which are usually multiple, with unclear margins, and have high risk of malignant transformation or recurrence.

Using lasers in combination with optical fibers, PDT has been used to manage oral precancerous and cancerous lesions, and the results are promising [22-24]. However, the high costs associated with the purchase, maintenance, and operation of lasers may become one of the biggest obstacles to the clinical acceptance of this new treatment. In addition, the laser system might not be necessary for superficial and wide-spread oral dysplasial lesions. To overcome the high costs of laser used in PDT, we developed a light-emitting diode (LED) light source. Furthermore, the sunny weather in Taiwan may worsen the unwanted systemic and cutaneous photosensitivity associated with PDT. To overcome the systemic side effect of cutaneous photosensitivity caused by ALA-PDT, we developed an ALA mucosal delivery system, which can localize ALA in lesion sites. We have found that this delivery system can enhance the efficiency of ALA-induced fluorescence in oral lesions (unpublished results). To evaluate the efficacy of ALA-PDT using the LED light source, the photodynamic effects were examined in cultured Ca9-22 gingival carcinoma cells, DMBA-induced dysplasial lesions on hamster buccal pouch carcinoma, and in clinical cases of oral leuokoplakia.

\section{MATERIALS AND METHODS}

\section{Cell Line}

Human gingival cancer (oral cavity squamous cell carcinoma) cell line Ca9-22 was obtained from JCRB cell bank. Stock culture of Ca9-22 cells was grown in Dulbecco's modified Eagle's medium supplemented with $10 \%$ fetal calf serum (FCS). Cell cultures were maintained at $37^{\circ} \mathrm{C}$ in a humidified atmosphere of $95 \%$ air and $5 \% \mathrm{CO}_{2}$. Antibiotics were not used routinely and Ca9-22 cells were found by routine testing to be negative for mycoplasma, bacteria, and fungi.

\section{Characterization of the LED Light Source}

For superficial and wide-spread oral dysplasial lesions, we designed a simple and inexpensive LED light source for
ALA-PDT. The light source consists of a high power LED with the wavelength centered at $635 \pm 5 \mathrm{~nm}$ and a bandwidth of $20 \mathrm{~nm}$ (Fig. 1A). The LED light source was fed by a small DC-power supply. Via optical lens, the LED light was coupled to an optical fiber with a diameter of $13 \mathrm{~mm}$. The irradiance intensity of LED device at the lesion surface was $100 \mathrm{~mW} / \mathrm{cm}^{2}$ with a spot size of $1 \mathrm{~cm}^{2}$ at a distance of $1.5 \mathrm{~cm}$ (Fig. 1B). The material cost of this LED light device is less than US $\$ 700$.

\section{ALA-PDT Induced Cytotoxicity on Ca9-22 Cells}

Ca9-22 cells were grown in 96-well plates at a density of $1.2 \times 10^{4}$ cells/well over night. The culture medium was removed and replaced with phenol red free DMEM medium (100 $\mu \mathrm{l} /$ well) containing $1 \mathrm{mM}$ ALA. The cells were incubated for 3 hours (avoiding light) and then irradiated with various doses of light using LED light source. For

A

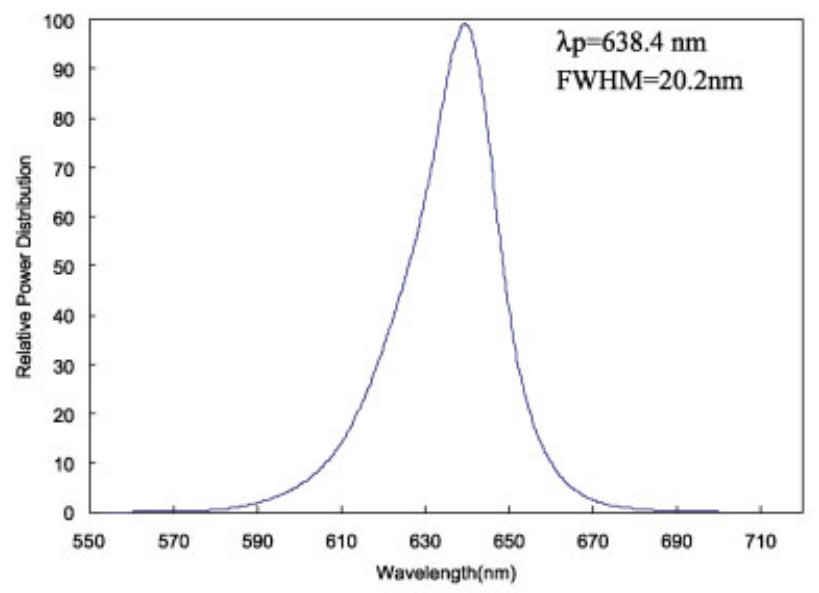

$\mathbf{B}$

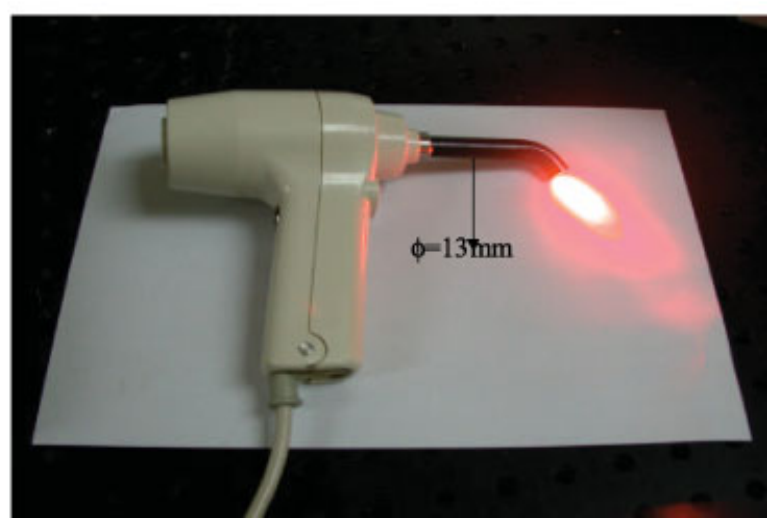

Fig. 1. A: The emission wavelengths of the Light-Emitting Diode (LED) light source are centered at $635 \pm 5$ with a bandwidth of $20 \mathrm{~nm}$. The light characteristics of LED are expressed as $\lambda p$ (peak maximum) and full width and half maximum (FWHM). The diameter of optical fiber is $13 \mathrm{~mm}$ $(\phi=13 \mathrm{~mm}) . \mathbf{B}$ : The appearance of the LED light source used in this study. 
comparison, we used a broadband lamp (VersaLight ${ }^{\mathbb{R}}$ ) developed by ESC Medical Systems Ltd., Israel. This light source used a superlight Xenon lamp fed by a high-power supply. The emitted light was filtered to yield a continuous spectrum of red light between $580-720 \mathrm{~nm}$ which could be used to excite cellular PpIX. The irradiance of filtered light at the surface was $100-150 \mathrm{~mW} / \mathrm{cm}^{2}$. In this study, the light intensity at the irradiance surface was fixed at $100 \mathrm{~mW} / \mathrm{cm}^{2}$. After light irradiation, the medium was replaced with DMEM containing 10\% FCS. Twenty-four hours later, cell survival was measured using an MTT [3(4,5-dimethyl-thiazoyl-2-yl) 2,5 diphenyl-tetrazolium bromide] assay.

MTT assay is based on the activity of mitochondria dehydrogenases, which can reduce a water-soluble tetrazolium salt to a purple insoluble formazan product [25]. The amount of MTT formazan product was analyzed spectrophotometrically at the absorbance of $570 \mathrm{~nm}$. Cells exposed to ALA but not light were used as control. Cell survival $(\%)=($ mean absorbance of treated cells/mean absorbance of control cells) $\times 100 \%$. Each individual phototoxic experiment was repeated for three times.

\section{Preparation of ALA Liquid}

The formulation of ALA was prepared using Pluronic F127 (BASF, Mount Olive, NJ) and Carbopol 971P which was kindly provided by BF Goodrich. Double concentrated gels of Pluronic F127 and Carbopol 971P in water were separately prepared prior to mixing. The "cold method" was adopted for preparing the Pluronic F127 gel [26]. After refrigerating at $4{ }^{\circ} \mathrm{C}$ overnight, clear solution form of Pluronic F127 was mixed with equal volume of the Carbopol gel, and the mixture was stored at $4^{\circ} \mathrm{C}$. Prior to use, $200 \mathrm{mg}$ of ALA was mixed with $800 \mathrm{mg}$ of the gel and packed into a 1-cc syringe. The formulation was used within 3 hours.

\section{ALA-Mediated PDT in DMBA-Induced Buccal Pouch Carcinoma Model}

Adult male (10-12-week-old) Syrian golden hamsters weighing 120-150 g were used for this study. A $0.5 \%(\mathrm{w} / \mathrm{v})$ solution of DMBA (7,12-dimethylbenzanthracene) in heavy mineral oil was applied thrice weekly to the left cheek pouches of all the animals $(n=3)$ in the experimental groups. At the end of 8 weeks, moderate or severe epithelial dysplasia was seen in the left cheek pouch [27]. For ALAPDT, ALA was topically applied onto the hamster cheek pouch with dysplasia. After 3 hours, $0.4 \mathrm{ml} 10 \%$ ketamine hydrochloride was given intramuscularly for anesthesia, the buccal pouch was pulled out, flattened, and irradiated with red light emitted from the LED light source. The irradiance intensity delivered to the pouch surface was $100 \mathrm{~mW} / \mathrm{cm}^{2}$. The light dose for the lesion was $100 \mathrm{~J} / \mathrm{cm}^{2}$. After macroscopic examination of the buccal pouch, the hamster was sacrificed 48 hours after light irradiation. The examined tissues were excised, fixed in $10 \%$ neutral buffered formalin, and embedded in paraffin. Sections of the tissue were stained with hematoxylin and eosin for histological examination in a standard manner.

\section{Patient and Lesions}

A total of 33 oral lesions were treated including leukoplakia (24), verrucous hyperplasia (5), erythroleukoplakia (2), and verrucous carcinoma (1) The represented case was a 40-year-old female patient having an oral dysplasia on left border of her tongue. She was treated in the Department of Oral and Maxillofacial Surgery, National Taiwan University Hospital. Written informed consent was obtained from the patient. This study was reviewed and approved by the Human Investigation Review Committee at the National Taiwan University Hospital. On day 1 of the treatment course, $20 \%$ of ALA liquid $\left(0.1 \mathrm{ml} / \mathrm{cm}^{2}\right)$ was applied to the leukoplakia lesion of the tongue for 2 hours and then activated with the LED light as described above. The irradiance intensity delivered to the mucosa surface was $100 \mathrm{~mW} / \mathrm{cm}^{2}$. The light dose for the treatment was $100 \mathrm{~J} /$ $\mathrm{cm}^{2}$. The treatment area includes the whole lesion with margins of normal tissue. Although there was mild burning sensation at the time of light illumination, she tolerated the treatment well and did not need anesthesia or analgesic medication. ALA-PDT was repeated twice in the say way on day 8 and day 15 , respectively.

\section{RESULTS}

\section{LED Array Is as Effective as VersaLight ${ }^{(\mathbb{R}}$ in ALA-PDT for Ca9-22 Cells}

The ability of ALA-PDT using LED light source to inhibit cell proliferation was initially tested on the human gingival cancer cell line Ca9-22. The photodynamic effects, measured by using an MTT assay, depended on the dose of light $(P<0.01$, multiple lineal regression) when the concentration of ALA was fixed to $1 \mathrm{mM}$. As shown in Figure 2, there was no statistically significant difference between the dose response curves of VersaLight ${ }^{\mathbb{R}}$ and LED while measured with the MTT assay ( $P>0.05$, multiple lineal regressions). The $\mathrm{LD}_{50}$ for VersaLight ${ }^{\circledR}$ and LED array were $4.5 \mathrm{~J} / \mathrm{cm}^{2}$ and $4.3 \mathrm{~J} / \mathrm{cm}^{2}$, respectively (Fig. 2).

\section{LED Array Is Effective in ALA-PDT for Oral Dysplasia in Hamsters}

To examine whether ALA-PDT could selectively destroy premalignant tissues with the LED light source, we established an oral dysplasia model in hamsters by repeated applications of DMBA to the mucosal surface of hamster buccal pouch for 8 weeks [27]. As shown in Figure 3A, epithelial dysplasia and chronic inflammation were found in the submucosa and muscle layer. After ALA-PDT using the LED light source, necrosis of the dysplasia lesions was noted (Fig. 3B). Epithelial necrosis was found in all the three cheek pouches receiving ALA-PDT 48 hours after exposure to the LED light. This result clearly indicates that LED light device can be used as the light source in ALA-PDT to destroy oral dysplasia lesions in hamsters.

\section{LED Array Is Effective in ALA-PDT for Treating Patients With Leukoplakia Lesion}

In the represented case, a white plaque is clearly visible and occupies a large area of the left lateral border of the 


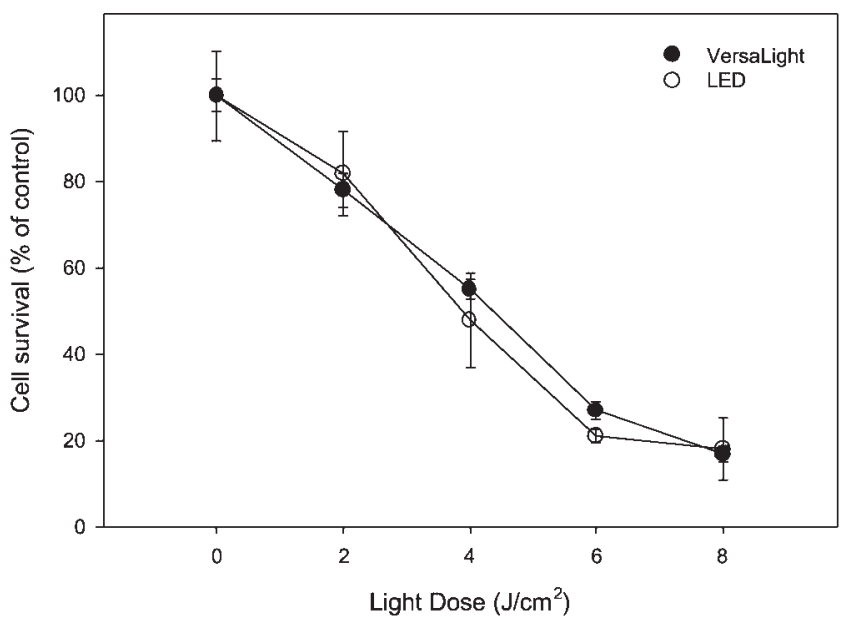

Fig. 2. 5-Aminolevulinic acid (ALA)-photodynamic therapy (PDT) induced cytotoxicity using different light sources. Ca922 cells were incubated with $1 \mathrm{mM}$ ALA and exposed to light irradiation at different light fluences. Cell viability was assessed by MTT assay 24 hours after light irradiation. Data are mean \pm SEM obtained from three independent experiments.

tongue (Fig. 4A). Microscopic picture of biopsy tissue from the leukoplakia revealed parakeratosis and acanthosis (Fig. 4B). Pseudohyphae of Candida albicans was also noted in the parakeratin layer (Fig. 4C). After ALA-PDT, the surface of the lesion became edematous and ulcerated, the edema disappeared after 24 hours, but the ulcer was still visible 1 week after treatment (Fig. 5A) and healed 1 week later (Fig. 5B). The leukoplakia disappeared completely without any scarring after three cycles of ALA-PDT (Fig. 5C). There is no recurrence of leukoplakia after followed up for 6 months. No cutaneous photosensitivity was noted during the course of treatment.

Using the LED light source, a total of 24 leukoplakia lesions were treated with ALA-PDT with complete responses in three, partial responses in nine, and no responses in twelve lesions as shown in Table 1 . In the five lesions of verrucous hyperplasia, complete responses were found in four while partial response in the remaining one. Partial responses were found in the two erythroleukoplakia and one verrucous carcinoma lesions.

\section{DISCUSSIONS}

PDT is a minimally invasive treatment available for palliation or eradication of several cancers. It can be applied alone or together with surgery, radiation therapy, or chemotherapy. Unlike radiotherapy, it can be repeated many times without cumulative toxicity. The preferential accumulation of PS in cancer cells combined with the localized delivery of light to the tumor, results in the selective destruction of the cancerous lesion. Compared to other traditional anti-cancer therapies, PDT does not involve generalized destruction of healthy cells. Besides, because PDT is a non-thermal process, it can preserve
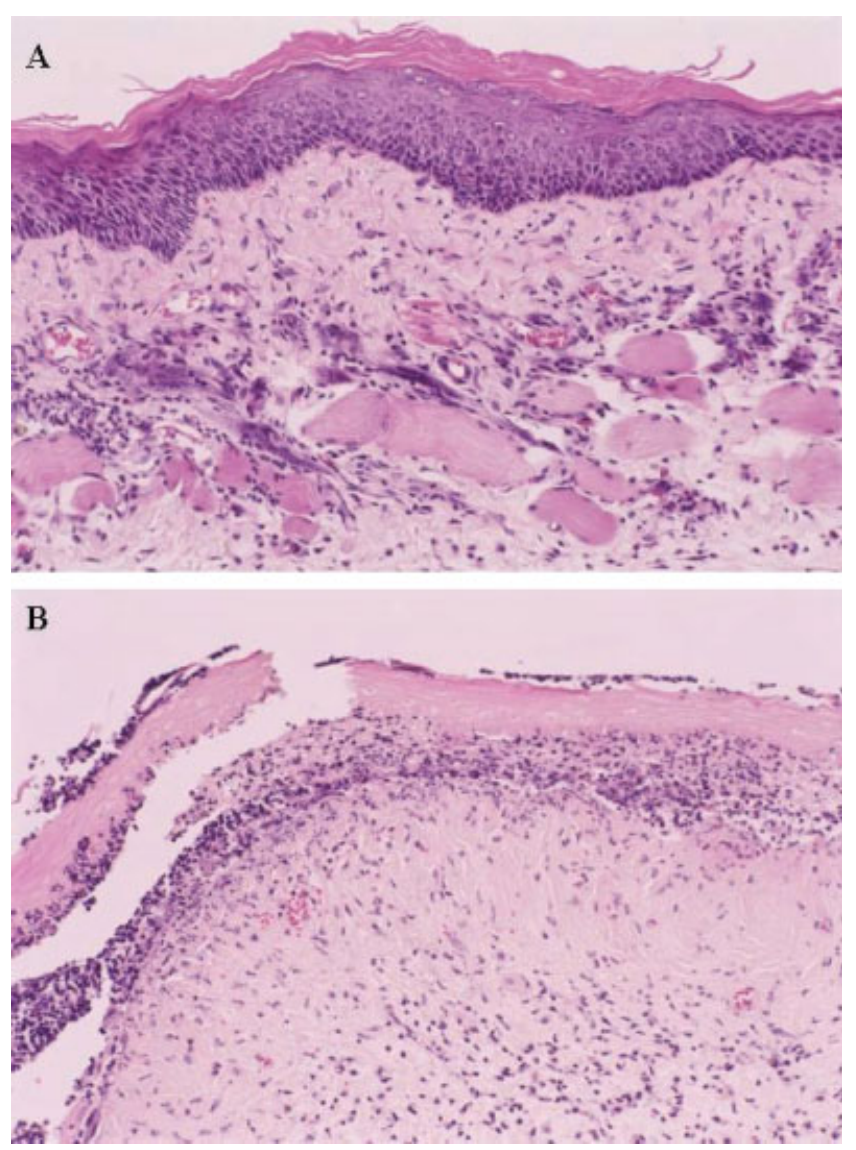

Fig. 3. Histological pictures of hamster buccal pouches 8 weeks after repeated application of DMBA, two days after LED light irradiation $\left(100 \mathrm{~J} / \mathrm{cm}^{2}\right)$. A: Control with light only. Epithelial dysplasia and chronic inflammation in the submucosa and muscle layer were noted in buccal pouch tissue without topical ALA application (hematoxylin and eosin stain, $\times 25$ ). B: ALA application followed by light irradiation. Epithelial necrosis and marked chronic inflammatory cell infiltration in the subepithelial connective tissue were noted (hematoxylin and eosin stain, $\times 25$ ).

connective tissues such as collagen and elastin, leaving little scarring and results in excellent cosmetic outcome. PDT is also valuable for premalignant conditions such as oral leukoplakia, mucosal dysplasia, or carcinoma in situ, as is shown in the representative case we present here. In this study, using the LED device, we found ALA-PDT effective in the cultured Ca9-22 gingival carcinoma cells and the DMBA-induced hamster buccal pouch carcinoma model. To evaluate its clinical efficacy for the treatment of oral neoplasia, we further apply ALA-PDT on patients having oral premalignant lesions. In the represented patient, the leukoplakia (Fig. 4), with pathological characteristics of parakeratosis and acanthosis, disappeared completely without any scarring after three cycles of ALAPDT using the LED light source (Fig. 5C). Complete responses were also found in patients with oral verrucous hyperplasia after ALA-PDT using LED light source. 

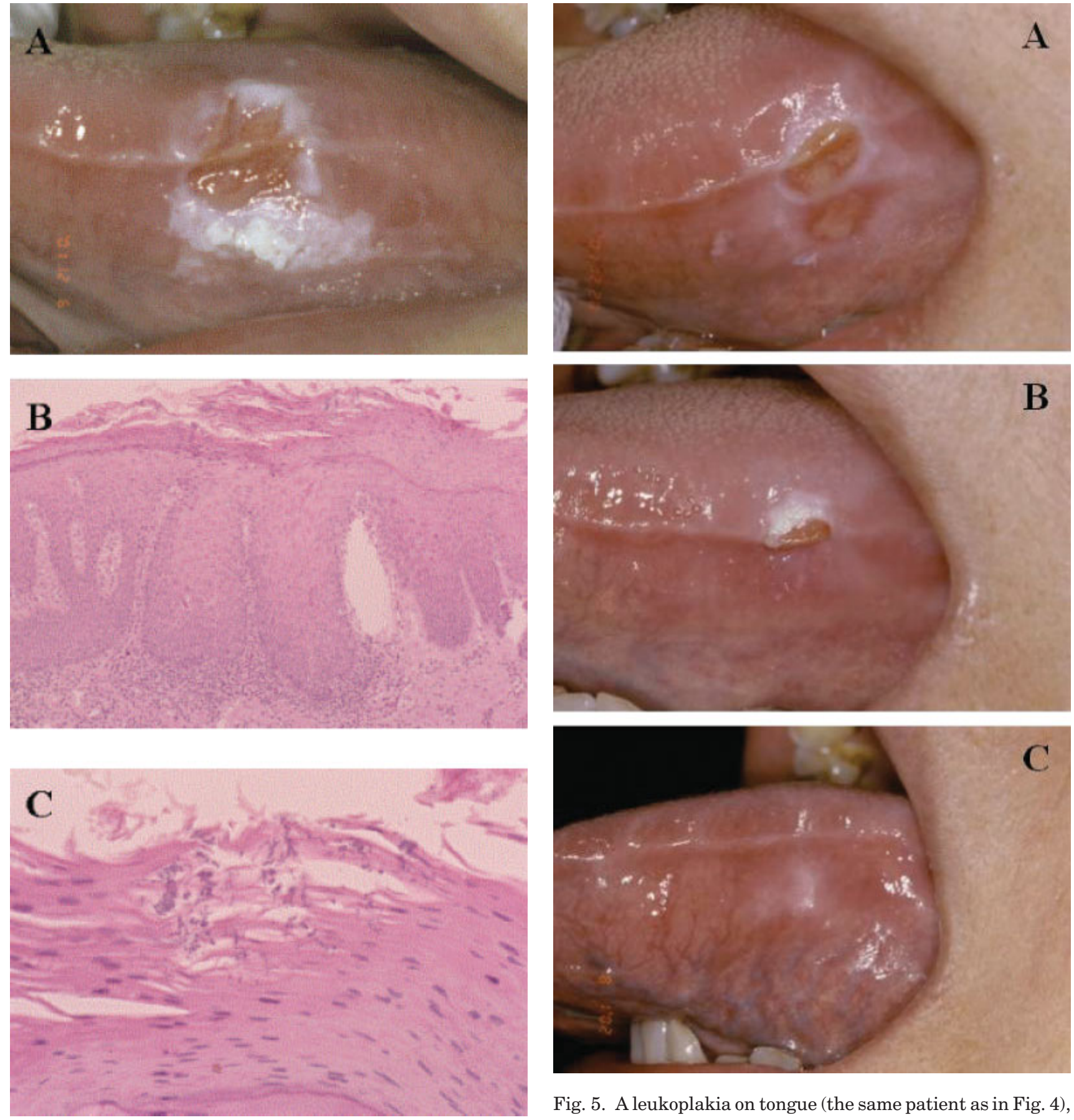

Fig. 4. A leukoplakia lesion on the tongue of a patient. A: The gross appearance showed that white plaque is clearly visible and occupies a large area of the left lateral border of the tongue; (B) Microphotographs of a biopsy specimen taken from the leukoplakia lesions showing parakeratosis and acnthosis (hematoxylin and eosin stain, $\times 25$ ); (C) Pseudohyphae of Candida albican were noted in the parakeratin layer (hematoxylin and eosin stain, $\times 25$ ).

Fig. 5. A leukoplakia on tongue (the same patient as in Fig. 4), treated with topical ALA application followed by LED irradiation $\left(100 \mathrm{~J} / \mathrm{cm}^{2}\right)$. A: One week after first ALA-PDT; (B) One week after second ALA-PDT; (C) One week after third ALAPDT. The leukoplakia improved gradually and disappeared finally after three times of ALA-PDT.

The three principle elements of PDT are oxygen, a PS, and light [28]. The commonly used PS are Photofrin ${ }^{\circledR}$ (profimer sodium), Levulan ${ }^{\circledR}$ (ALA), Visudyne ${ }^{\text {TM }}$ (Verteprofin/BPD, benzoporphyrin derivative), and Foscan ${ }^{\mathbb{R}}$ (m-THPC; meta-tetrahydroxyphenyl chlorine). They have 
TABLE 1. Results of ALA-PDT for the Treatment of Oral Dysplasial Lesions

\begin{tabular}{lccccc}
\hline Oral lesions & Total & Complete response & Partial response & No response & Follow-up (months) \\
\hline Leukoplakia & 24 & 3 & 9 & 12 & 6 \\
Erythroleukoplakia & 2 & & 2 & 6 \\
Verrucous hyperplasia & 5 & 4 & 1 & 6 \\
Verrucous carcinoma & 1 & 7 & 13 & 6 \\
Total & 32 & 7 & 12 & \\
\hline
\end{tabular}

received approval by regulatory authorities, but are quite expensive. The most convenient and controllable light source for PDT are Lasers. Lasers provide a monochromatic, very bright light that can reduce the time necessary to deliver the final PDT dose. In addition, the coherent property of laser makes it very efficiently coupled to an optical fiber. In this way, lesions in deep-seated tissues can be illuminated effectively. The choice of laser wavelength is crucial since it must be matched with the often narrow absorption band of the PS. Presently, diode laser systems are used clinically for PDT. Compared to other laser systems, diode lasers are less expensive, easy to operate, and portable for clinical use. Due to the technical reasons, diode lasers can only offer a single output wavelength. Therefore, one laser can only be used in combination with one PS. For example, the diode laser used clinically for Photofrin ${ }^{\circledR}$ PDT was fixed at $630 \mathrm{~nm}$. In addition to the high cost associated with PS, the costs of purchase, maintenance, and operation of lasers are still high too, and thus, may become one of the biggest obstacles for PDT to be widely accepted clinically.

Non-laser light sources (tungsten, xenon arc, metal halide, fluorescent, etc.) have also been employed to perform PDT. Lamps provide a broad range of wavelengths at reduced fluence rates. Since most investigators use light with relatively low fluences $\left(100-300 \mathrm{~mW} / \mathrm{cm}^{2}\right)$ to avoid thermal effects, the use of lamps does not necessarily produce a dramatic increase in the time required for the treatment. Because of their broad emission, lamps can be used in combination with several PS with different absorption maximum within the emission spectrum of the lamp. So, the same lamp could be used for PDT with Foscan ${ }^{\circledR}$, Photofrin, or ALA. The availability of broadband sources is challenging the use of lasers where light can be directly delivered to the tumor without coupling to an optical fiber such as tumors of skin, head and neck, and oral cavity.

In the past few years, the development of high power LED has advanced them to a stage where their use in PDT is possible. LED would offer several advantages for clinical use. The bandwidth is $5-20 \mathrm{~nm}$ and the power output can be as high as $150 \mathrm{~mW} / \mathrm{cm}^{2}$ over an area of approximately $20 \mathrm{~cm}^{2}$. The power output can still be a limiting factor in their wide-spread use for PDT, however further improvement in their technology could improve this aspect. The major characteristics in favor of LED used for PDT are price and versatility. LED is inexpensive (in compar- ison with all the other sources described so far) and can be arranged in array to irradiate large areas. Moreover, they can be arranged in different geometric combination to compensate for difficult anatomic areas. To choose a light source for PDT, reliability, ease of use, cost, and space are the most important variables that need to be considered in a clinical setting.

It has been reported that the optimum wavelength for ALA-PDT is actually around $635 \mathrm{~nm}$ [29]. In this study, the LED used is suitable for ALA-PDT because the wavelength of LED was centered at $635 \pm 5 \mathrm{~nm}$. Compared to other laser or non-laser light sources, this LED device was not only effective but also cheaper for ALA-PDT. It is safe, convenient, and easy to use in the treatment of oral leukoplakia or dysplasia (mild, moderate, and severe). The portability and ease of use of the LED light source appear to make it very attractive for superficial treatment. Illumination using the LED light source at a defined wavelength with a relatively narrow spectral bandwidth allows accurate light dosimetry at the surface of the lesion. If a broadband source is used, the depth of light penetration, the extinction coefficient of the sensitizer and the spectral intensity of the illumination can all vary across the bandwidth of light used [30].

Unlike the other PS, ALA can be administered topically and orally and is the preferred choice for superficial lesions in skin and oral cavity [31-33]. There is growing evidence that topical application of ALA is as effective as systemically administered 5-ALA, resulting in a high response rate, with excellent healing and little to no scarring of the treated site. Our present study indicates that topical ALA application together with a convenient and easy-to-use LED light source is an attractive development for the treatment of oral dysplasia. 5-ALA is eliminated rapidly from the body, so that treatment can take place on an outpatient basis without the risk of cutaneous photosensitivity, while appropriately trained nursing staff can use the LED light source safely. With the LED light source, PDT can emerge as an attractive option for mucosal dysplasia and carcinoma in situ patients, because a large area of mucosa, including areas of apparently normal mucosa, can be treated superficially.

\section{ACKNOWLEDGMENTS}

The authors thank Yu-I Lin for her assistance on the experiment. 


\section{REFERENCES}

1. Cancer registry annual report in Taiwan area. Department of Health, The Executive Yuan, Taiwan, 2001.

2. Ho PS, Ko YC, Yang YH, Shieh TY, Tsai CC. The incidence of oropharyngeal cancer in Taiwan: An endemic betel quid chewing area. J Oral Pathol Med 2002;31:213-219.

3. Ko YC, Huang YL, Lee CH, Chen MJ, Lin LM, Tsai CC. Betel quid chewing, cigarette smoking and alcohol consumption related to oral cancer in Taiwan. J Oral Pathol Med 1995; 24:450-453.

4. Kwan HW. A statistical study on oral carcinomas in Taiwan with emphasis on the relationship with betel nut chewing: A preliminary report. Taiwan I Hsueh Hui Tsa Chih 1976;75: 497-505.

5. Chen YK, Huang HC, Lin LM, Lin CC. Primary oral squamous cell carcinoma: An analysis of 703 cases in southern Taiwan. Oral Oncol 1999;35:173-179.

6. Silverman S Jr, Gorsky M, Lozada F. Oral leukoplakia and malignant transformation. A follow-up study of 257 patients. Cancer 1984;53:563-568.

7. Lee CH, Ko YC, Huang HL, Chao YY, Tsai CC, Shieh TY, Lin LM. The precancer risk of betel quid chewing, tobacco use and alcohol consumption in oral leukoplakia and oral submucous fibrosis in southern Taiwan. Br J Cancer 2003;88:366-372.

8. Lippman SM, Benner SE, Hong WK. Cancer chemoprevention. J Clin Oncol 1994;12:851-873.

9. Henderson BW, Dougherty TJ. How does photodynamic therapy work? Photochem Photobiol 1992;55:145-157.

10. Dolmans DE, Fukumura D, Jain RK. Photodynamic therapy for cancer. Nat Rev Cancer 2003;3:380-387.

11. Weishaupt KR, Gomer CJ, Dougherty TJ. Identification of singlet oxygen as the cytotoxic agent in photoinactivation of a murine tumor. Cancer Res 1976;36:2326-2329.

12. Foote CS. Definition of type I and type II photosensitized oxidation. Photochem Photobiol 1991;54:659.

13. Dougherty TJ, Gomer CJ, Henderson BW, Jori G, Kessel D, Korbelik M, Moan J, Peng Q. Photodynamic therapy. J Natl Cancer Inst 1998;90:889-905

14. Peng Q, Berg K, Moan J, Kongshaug M, Nesland JM. 5Aminolevulinic acid-based photodynamic therapy: Principles and experimental research. Photochem Photobiol 1997;65: 235-251.

15. Gardner LC, Smith SJ, Cox TM. Biosynthesis of deltaaminolevulinic acid and the regulation of heme formation by immature erythroid cells in man. J Biol Chem 1991;266: 22010-22018

16. Kennedy JC, Pottier RH. Endogenous protoporphyrin IX, a clinically useful photosensitizer for photodynamic therapy. J Photochem Photobiol B 1992;14:275-292.

17. Messmann H, Mlkvy P, Buonaccorsi G, Davies CL, MacRobert AJ, Bown SG. Enhancement of photodynamic therapy with 5-aminolaevulinic acid-induced porphyrin photosensitisation in normal rat colon by threshold and light fractionation studies. Br J Cancer 1995;72:589-594.

18. Friesen SA, Hjortland GO, Madsen SJ, Hirschberg $\mathrm{H}$, Engebraten O, Nesland JM, Peng Q. 5-Aminolevulinic acidbased photodynamic detection and therapy of brain tumors. Int J Oncol 2002;21:577-582.
19. Soler AM, Angell-Petersen E, Warloe T, Tausjo J, Steen HB, Moan J, Giercksky KE. Photodynamic therapy of superficial basal cell carcinoma with 5-aminolevulinic acid with dimethylsulfoxide and ethylendiaminetetraacetic acid: A comparison of two light sources. Photochem Photobiol 2000; 71:724-729.

20. Mlkvy P, Messmann H, Regula J, Conio M, Pauer M, Millson CE, MacRobert AJ, Bown SG. Photodynamic therapy for gastrointestinal tumors using three photosensitizersALA induced PPIX, photofrin and mTHPC. A pilot study. Neoplasma 1998;45:157-161.

21. Kennedy JC, Marcus SL, Pottier RH. Photodynamic therapy (PDT) and photodiagnosis (PD) using endogenous photosensitization induced by 5 -aminolevulinic acid (ALA): Mechanisms and clinical results. J Clin Laser Med Surg 1996;14 289-304.

22. Nauta JM, van Leengoed HL, Star WM, Roodenburg JL, Witjes MJ, Vermey A. Photodynamic therapy of oral cancer A review of basic mechanisms and clinical applications. Eur J Oral Sci 1996;104:69-81.

23. Fan KF, Hopper C, Speight PM, Buonaccorsi G, MacRobert AJ, Bown SG. Photodynamic therapy using 5-aminolevulinic acid for premalignant and malignant lesions of the oral cavity. Cancer 1996;78:1374-1383.

24. Biel MA. Photodynamic therapy and the treatment of head and neck neoplasia. Laryngoscope 1998;108:1259-1268.

25. Mosmann T. Rapid colorimetric assay for cellular growth and survival: Application to proliferation and cytotoxicity assays. J Immunol Methods 1983;65:55-63.

26. Schmolka JR. Artificial skin. I. Preparation and properties of pluronic F-127 gels for treatment of burns. J Biomed Mater Res 1972;6:571-582.

27. Chen C-T, Chiang HHK, Chow S-N, Wang C-Y, Lee Y-S, Tsai J-C, Chiang C-P. Autofluorescence in normal and malignant human oral tissues and in DMBA-induced hamster buccal pouch carcinogenesis. J Oral Pathol Med 1998; 27:470-474

28. Hopper C. Photodynamic therapy: A clinical reality in the treatment of cancer. Lancet Oncology 2000;1:212-219.

29. Moan JK, Berg XX, Iani V. Action spectra of dyes relevant for photodynamic therapy. In: Moser JG, editor. Photodynamic Tumor Therapy. Amsterdam: Harwood Academic Publishers; 1998:169-181.

30. Stringer MR. Problems associated with the use of broad-band illumination sources for photodynamic therapy. Phys Med Biol 1995;40:1733-1735.

31. Gerscher S, Connelly JP, Griffiths J, Brown SB, MacRobert AJ, Wong G, Rhodes LE. Comparison of the pharmacokinetics and phototoxicity of protoporphyrin IX metabolized from 5-aminolevulinic acid and two derivatives in human skin in vivo. Photochem Photobiol 2000;72:569-574.

32. Kubler A, Haase T, Rheinwald M, Barth T, Muhling J. Treatment of oral leukoplakia by topical application of 5aminolevulinic acid. Int J Oral Maxillofac Surg 1998;27:466469.

33. Rhodes LE, Tsoukas MM, Anderson RR, Kollias N. Iontophoretic delivery of ALA provides a quantitative model for ALA pharmacokinetics and PpIX phototoxicity in human skin. J Invest Dermatol 1997;108:87-91. 